\title{
LOS VALORES QUE CIMENTAN \\ LA IDENTIDAD Y EL \\ COMUNITARISMO
}

Ana Teresa López de Llergo

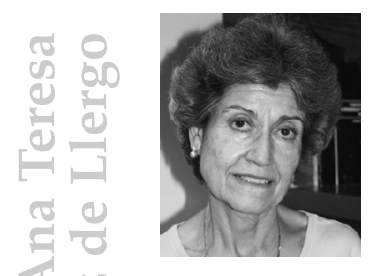

Doctorado en Ciencias de la Educación, Universidad de Navarra. Maestra en Pedagogía, Universidad Panamericana.

Licenciada en Ingeniería Química y Química Industrial, Universidad Iberoamericana.

Directora de Difusión Cultural, Universidad Panamericana, sede México.

Docente e investigadora, Facultad de Pedagogía, Universidad Panamericana, sede México.

Ponente en Congresos nacionales e internacionales. Autora de: La empresa y la promoción de valores, Ed. UP-Facultad de Filosofía; Hacia un desarrollo humano, Ed. Limusa; Valores, valoraciones y virtudes, Ed. CECSA.

Correo electrónico: [alopezde@up.edu.mx].

\section{RESUMEN}

En este trabajo se aborda el tema de la identidad entendida como la percepción del ser humano sobre sí mismo, y el comunitarismo como la conciencia de pertenecer a un grupo. Estos dos aspectos constitutivos de la dinámica de la personalidad encuentran su raíz en los valores. La identidad en la intimidad, la comunicabilidad y la libertad. El comunitarismo en la solidaridad, la subsidiariedad, el respeto y la lealtad. Sin embargo, los valores no bastan: es preciso vincularlos con las virtudes sociales.

Palabras clave: Identidad; comunitarismo; valores; virtudes; intimidad; comunicabilidad; libertad; solidaridad; subsidiariedad; respeto; lealtad; prudencia; justicia; fortaleza; templanza. 


\begin{abstract}
In this project, we approach the topic of identity, understood as the perception of the Human Being on himself, and the Communitarism as the consciuosness of belonging to a group. These two constitutive aspects of the personality dynamics find their roots in values. Identity in intimacy, communicability and freedom. Communitarism in solidarity, subsidiarity, respect and loyalty. However, values are not enough, it is necessary to join them with social virtues.
\end{abstract}

Key words: Identity; communitarism; values; virtus; intimacy; communicability; liberty; solidarity; subsidiarity; respect; loyalty; prudence; justice; fortress; moderation.

\title{
LA IDENTIDAD
}

El ser humano al ser individuo y miembro de una sociedad ha de equilibrar dos tendencias constitutivas: la identidad como autoconocimiento y autoaceptación, y el comunitarismo como sentido de pertenencia y colaboración dentro de la sociedad.

La identidad manifiesta lo que cada uno es. La posibilidad de descubrir la identidad se debe al ejercicio de la conciencia vuelta sobre sí y a la asunción de los sentimientos que tal conocimiento desata. Es un proceso con dos niveles: el intelectual donde se descubre y se reconoce la propia realidad (comúnmente denominado autoconocimiento), y el afectivovolitivo que consiste en admitir los límites y las posibilidades personales (esto es la autoaceptación).

Conocer es una apropiación inmaterial del objeto de conocimiento. El conocimiento con el más alto grado de espiritualidad es el que se refiere a sí mismo. El autoconocimiento implica la detección de la variada geografía interior: sentimientos, afectos, gustos, tendencias, ilusiones, proyectos, cualidades y defectos. Y, también, incorpora el entorno familiar y social, el estado civil y la profesión, los recursos y la capacidad de relación... El conocimiento propio se enriquece cuando se admite el conocimiento que los demás tienen de uno; ellos cuentan con una perspectiva inalcanzable para quien se está descubriendo. 
La autoaceptación se expresa con una postura realista y equilibrada para admitir lo que se es sin ocultar algunos aspectos o añadir lo que no se posee. Ayuda a asumir con serenidad y optimismo las fortalezas y debilidades y, sobre todo, a querernos como somos. Todo ello aporta una base objetiva para crear proyectos de superación, y así, el proceso va desde un nivel en el que nos queremos, a otro en el que nos queremos más porque logramos los propósitos establecidos. Es importante apoyarse en los valores para evitar actitudes evasivas que deterioran la aceptación de la propia identidad, con las consecuencias de inadaptación, inseguridad, aislamiento y, en el peor de los casos, de agresividad. Cuando existe autoaceptación es segura la oportunidad para ocupar el sitio correspondiente y desempeñar el papel debido. Se trata de alguien bien ubicado en el mundo y capaz de realizar su proyecto de vida cimentado en la posibilidad.

\section{EL COMUNITARISMO}

Es el resultado de una actitud social ${ }^{1}$ que facilita la suma de los esfuerzos de los pobladores de una región, de los grupos intermedios - escuelas, empresas, clubs, etcétera- y del gobierno, para mejorar las condiciones económicas, sociales y culturales de las comunidades. Para ello, se trabaja en diagnósticos, planes, ejecuciones y evaluaciones de programas y proyectos. El impulso al comunitarismo se visualiza como una manera de sensibilizar y motivar a las personas para participar en la solución de sus problemas. Tiende al mejoramiento colectivo de la comunidad a través de la promoción individual y solidaria de sus integrantes para lograr un fin común. Su actividad es multifacética y se expresa en programas cuya meta es alcanzar mejoras concretas. Más que una acción sobre la comunidad, es una acción de la comunidad. Se trata de esfuerzos y acciones de base, organizadas con la iniciativa y dirección de los mismos involucrados, aunque para su eficacia se recurra a agentes externos, y durante su ejercicio, se prevea asistencia técnica.

Cfr. LÓPEZ DE LLERGO, A.T. y CRUZ DE GALINDO, L. M., «Desarrollo comunitario y calidad de vida»., Revista Panamericana de Pedagogía. Saberes y quehaceres del pedagogo., n. 4 , p. $60-64$. 
El comunitarismo no homogeniza a los individuos: cada uno tiene sus intereses particulares, pero son capaces de asumir otros a nivel común. Gracias al sentido de identidad y pertenencia, los diversos sujetos orientan sus personales intereses al logro de intereses colectivos. El trabajo comunitario es trabajo con grupos unidos por objetivos y con doble esfuerzo, uno buscará alcanzar las metas propuestas, el otro, neutralizar las tendencias individualistas.

El comunitarismo asume que las comunidades pueden desarrollar la capacidad para resolver sus problemas: las personas quieren y pueden cambiar de una óptica individualista a la comunitaria, esto es, de los enfoques fragmentados a los holísticos, de la exclusión a la integración, de la indiferencia a la cooperación. Lo imprescindible es identificar la responsabilidad personal con la responsabilidad social, sin que desaparezcan ninguna de las dos. Así, el desarrollo comunitario se llevará a cabo en una atmósfera de respeto a los valores del contexto cultural, ya sea a nivel regional, nacional o internacional. En el siguiente texto queda claro que el discurso no es el de la ciudadanía sino el de la civilidad, no es el de la recepción pasiva sino el de la participación activa propia de la actitud comunitaria.

[...] el comunitarismo es el paraguas más adecuado para entender las relaciones sociales como el marco en el que operan una pluralidad de sujetos y no sólo de individuos y estado. Por otro lado, ni la ciudadanía es aquello que se predica del ciudadano, ni la civilidad es lo que se dice de la relación que conforma ámbitos sociales ${ }^{2}$.

El compromiso personal con la comunidad y el reconocimiento de la propia identidad con la inclusión de todos los ámbitos - familia, sociedad, instituciones, patria y naciones vecinas- propician el sentido de pertenencia. Como es imposible vivir en un medio totalmente adecuado, el ser humano lo modifica de acuerdo a sus requerimientos; esto es mucho más valioso que un simple proceso de adaptación. Al realizar este esfuerzo, el entorno adopta un rostro familiar para quienes lo transforman y, por eso,

2 PÉREZ ADÁN, J., La comunidad educativa., p. 182. 
se establece una relación más estrecha. El medio se convierte en una prolongación de la persona debido a la huella de su trabajo.

El sentido de pertenencia consiste en el arraigo de cada uno con los ámbitos donde se desenvuelve. Aquí aparece una doble vertiente: la primera es la toma de conciencia de la necesidad de esa vinculación, y la segunda es la dimensión afectiva donde se asume la precariedad de toda persona y el modo de compensarla con la ayuda mutua. Pero en donde se manifiesta la nobleza de cada persona es en la inquietud por retribuir, aportando lo que uno es capaz de proporcionar como reciprocidad a lo recibido. Para facilitar el sentido de pertenencia se ha de lograr que el ambiente sea hospitalario y seguro.

Lo opuesto al sentido de pertenencia es el desarraigo, la ausencia de raíces. El desarraigado destruye socialmente, por ausencia de interacción. Las manifestaciones de desarraigo son variadas y a veces se ocultan tras una desmedida defensa de la propia libertad, unida a la notable desconsideración por los demás.

\section{Propuesta de Valores ${ }^{3}$ que Cimientan la Identidad y el Comunitarismo}

\section{a) Valores que cimientan la identidad}

Los valores que dan cimiento a la identidad forman parte de los constitutivos intrínsecos de todo ser humano son la intimidad, la comunicabilidad y la libertad ${ }^{4}$. Estos tres valores son notas características que gracias a la espiritualidad también son valores genéricos de toda persona. La intimidad se refiere a la autoconciencia y a la riqueza interior, y explica la fortaleza

3 El concepto de valor es: toda perfección real o posible que procede de la naturaleza y se apoya tanto en el ser como en la razón de ser de toda realidad. Esta perfección merece nuestra estima reconocimiento y agrado. (LÓPEZ DE LLERGO, A.T., Valores, valoraciones y virtudes, p. 34).

4 Cfr. LÓPEZ DE LLERGO, A.T., Educación en valores, educación en virtudes., p. 49-51. 
radical de los seres humanos. En este valor, como en los demás, se apoyan las virtudes, que cuando se adquieren, ofrecen especial solidez a la persona, tanto para acometer como para resistir, pero el valor de la intimidad hace que cada virtud posea en cada cual un matiz inédito. Aquilino Polaino-Lorente y Pedro Martínez Cano señalan: «La persona es una realidad de la que brotan novedades, una intimidad creativa, capaz de crecer» ${ }^{5}$. En la intimidad anida la voz de la conciencia. Por eso no se puede hablar de intimidad con uno mismo sin tener en cuenta la presencia de una voz propia, que parece tener como finalidad emitir juicios últimos especialmente sobre realidades últimas, y en términos de bien y mal. Por lo tanto, el ámbito de la intimidad es, entre otras cosas, un reducto moral, donde se emiten juicios de aprobación y condena, donde surgen sentimientos de paz y tranquilidad, y de remordimiento y de culpa.

La comunicabilidad es un valor que apoya los valores relacionales. Todo lo que uno posee en su intimidad - por aprendizaje o por creación imaginativa - tiende a manifestarse. La persona es un ser que se muestra a los demás; puede decidir si exterioriza o no lo que posee en su interior. El valor de la comunicabilidad es motor de su comportamiento ${ }^{6}$. La comunicabilidad ayuda a jerarquizar y a relacionar personas, cosas y distintas actividades, para promover con eficacia la armonía individual y social. Gracias a este valor, los seres humanos pueden compartir lo propio y lo de los demás, para un mejor aprovechamiento de recursos.

La libertad es un valor propio y ajeno. Por ella el ser humano vive y se realiza libremente poseyéndose y siendo dueño de sus actos. En cada acto libre cada quien está en sí mismo todo él, allí se asume, se sintetiza y se puede entregar, de este modo el don personal es regalo y no arrebato. Todo ello manifiesta la capacidad de sacar de sí para dar, pero no hay dar sin aceptar y, en ese momento se establece el diálogo con la libertad del otro $^{7}$. La actividad del ser humano generalmente se apoya en la elección.

MARTÍNEZ CANO, P., La comunicación en la pareja., p. 24.

Cfr. Ibidem., p. 25.

Cfr. Idem. 
En la persona, el aprendizaje es en algunos aspectos mucho más importante que el instinto, la actividad mecánica o la intuición ${ }^{8}$. Por eso, la vida humana no es automática, cada cual tiene la posibilidad de participar en los avances, pero contando con que el éxito no está asegurado.

La libertad necesita una trama común, que podríamos definir como fortalecimiento de los derechos humanos. El concepto de libertad reclama, por su misma esencia, un complemento que le proporcionan estos dos nuevos conceptos: lo justo y lo bueno. Podríamos decir que es propia de la libertad la capacidad de la conciencia para percibir los valores humanitarios fundamentales que atañen a todos los hombres ${ }^{9}$. La persona no puede y no debe ser instrumentalizada por las estructuras sociales, económicas y políticas, porque todo ser humano posee la libertad de orientarse a su fin último. Por otra parte, toda realización cultural, social, económica y política — en la que actúa la sociabilidad de la persona y su actividad transformadora- es transitoria, pueden surgir otras opciones, por eso, ningún planteamiento tiene legitimidad para oprimir ${ }^{10}$.

\section{b) Valores que cimientan el comunitarismo}

Los valores que dan cimiento al comunitarismo son los valores relacionales: la solidaridad, la subsidiariedad, el respeto y la lealtad ${ }^{11}$.

Existe una gran variedad de valores en el ámbito social, pero nos referiremos a los valorse de la solidaridad, la subsidiariedad, el respeto y la lealtad porque tienen capacidad de sustentar a los demás. El valor de la solidaridad fortalece la unidad ciudadana, pues integra a los miembros del colectivo. Por este valor se manifiestan los de cada ciudadano, se evita que algunas personas se aíslen, y se mejora la sociedad al contar con más

8 Cfr. YEPES STORE, R. y ARANGUREN ECHEVARRÍA, J., Fundamentos de Antropología. Un ideal de la excelencia humana., p. 24 y ss.

9 RATZINGER, J., Verdad, valores, poder., p. 33-34.

${ }^{10}$ Cfr. Compendio de la doctrina social de la Iglesia., n. 48.

${ }^{11}$ Cfr. LÓPEZ DE LLERGO, A.T., Educación en valores, educación en virtudes., p. 54-56. 
recursos que favorecen el progreso y el desarrollo. Cada uno aporta lo propio. Es un valor que evita la indiferencia y la apatía. La solidaridad es sumamente necesaria para neutralizar la competitividad malsana, fenómeno cotidiano y omnipresente en todos los terrenos de la existencia, germen de insociabilidad, de cerrazón individualista y de desprecio.

El valor de la subsidiariedad también logra que efectivamente haya otro tipo de colaboración. La diferencia con la colaboración solidaria consiste en que aquí se detectan las carencias de los demás y se cubren con las capacidades propias, se ejercita la comprensión y la misericordia, el compromiso es más generoso porque se dona más de lo que se recibe, se comparte. El subsidio expresa la urgencia de colocar al otro en condiciones de mostrar su dignidad y, si temporalmente no tiene la capacidad de subsistir dignamente, se le ofrecen los medios hasta que vuelva a ser autosuficiente. La práctica de este valor forma el tejido social y constituye la base de una verdadera comunidad de personas. De este modo es posible el reconocimiento de formas más elevadas de sociabilidad, pues aquí hay mucho más que la simple justicia: hay regalo.

El valor del respeto consiste en el trato interpersonal apoyado en el reconocimiento de que toda persona posee la misma dignidad y, por eso, es sujeto de derechos humanos. Además, la innegable variedad de modos de ser, de modos de reaccionar, de modos de resolver los asuntos, de modos de manifestar la intimidad, de modos de cuidar, son ejemplo y cauce para diseñar la propia actividad; por eso, todos nos debemos mutuamente respeto, pues de todos aprendemos. También merece nuestro respeto el entorno natural, marco de la vida humana, que debe estar al servicio de los seres humanos. El respeto facilita la convivencia.

El valor de la lealtad es indispensable para la cohesión y la comprensión. Este valor afina la sensibilidad para reconocer la mutua vinculación. Es un valor profundo que excede a las relaciones de justicia, porque en la lealtad hay gratuidad y todos buscan la integridad personal, social - familiar-o de las propiedades. La lealtad proporciona fundamento a la honradez, sólo así se hace posible este valor, tan precario en 
nuestro tiempo por haberlo cimentado en meras formas externas. La práctica de la lealtad garantiza relaciones estables: cada uno se hace acreedor de confianza. La confianza es indispensable en las auténticas, sólidas y estables relaciones humanas.

La actitud de civilidad consistirá en descubrir y promover los valores de los miembros de la sociedad — familiar, laboral, recreativa- para animarlos a que sus relaciones los manifiesten. En el seno familiar se favorece el conocimiento de los individuos, facilitando la promoción de los valores sociales y el impulso de las buenas relaciones naturales de los consanguíneos entre sí, y con el entorno social. En la vida social existen instituciones que se organizan para resolver distintas necesidades. Sin embargo, como ya se señaló, no es el individuo quien ha de subordinarse totalmente a la sociedad, sino que esta última ha de agotar su dinamismo en la persona, pues los valores sociales dependen de quienes los transmiten. La sociedad ha de propiciar esa transmisión por medio de un buen ambiente, lo que favorece la salud social.

Algunos problemas para alcanzar valores en la sociedad se deben al pluralismo de ideas y no siempre quienes están más preparados tienen las ideas más visionarias. El recelo ante los valores ocasiona relativismos axiológicos y ello conduce a una mal entendida democracia. También causa problemas el materialismo axiológico que suprime los valores espirituales.

Estas connotaciones influyen de manera evidente en el planteamiento democrático, donde se abre a todos la posibilidad de participación, aspecto en general muy positivo. Sin embargo, el problema aparece cuando no se considera el diferente peso de las aportaciones. Muchas veces se toman más en cuenta las de quienes las exponen de forma más deslumbrante — sagacidad, agilidad, simpatía, etcétera- que aquellas que parecen tediosas por su profundidad y constancia. Además, la tendencia general en nuestro mundo globalizado, promueve una tolerancia donde no se contemplan los valores absolutos por el temor de favorecer más a algunas culturas, con lo cual las estructuras se aplanan y la axiología se relativiza. También el peso que se proporciona a la evidencia física es contundente y la realidad espiritual muchas veces queda 
asumida por la materia, otras veces negada, o en el mejor de los casos, diluida y sin influencia vital.

Con motivo de la reunión del CELAM, en Brasil, en mayo de 2007, Benedicto XVI detectó los siguientes problemas que obstaculizan la democracia en América Latina. Uno es el peligro de gobiernos autoritarios o sujetos a ideologías supuestamente superadas. También, el peligro de que la economía liberal olvide la equidad, pues siguen aumentando sectores sociales en extrema pobreza e incluso excluidos de los propios bienes naturales. La educación es la respuesta que siempre ofrecemos a la solución de los problemas. Sin embargo, esto que está a flor de piel, esta evidencia, en la práctica no está resuelta, posiblemente porque los planes de acción educativa no están bien fundamentados. La educación se acomoda mal a la investigación, a las demandas, al análisis de la realidad, a la consideración de todas las variables. Por eso, no se han encontrado las teorías educativas válidas que sustenten las prácticas en el aula de clases o en los ambientes educativos ${ }^{12}$.

\section{La Participación Comunitaria en lo Local y en lo Global con Base en Valores Relacionales y en Virtudes ${ }^{13}$ Sociales}

Cuando las personas se integran comunitariamente a su entorno próximo, cuando su identidad está profundamente vinculada a lo local y sienten el compromiso, la responsabilidad y la lealtad de beneficiar, están en mejores condiciones de abrirse a un entorno más amplio, diverso en costumbres, en recursos, en estilos de vida. La solidez de asentarse en lo local para aportar a lo global, es posible gracias a un humanismo axiológico que podrá animar un nuevo orden social, económico y político, fundado sobre la dignidad y la libertad de toda persona humana, y que actúa a través de los valores relacionales y de las virtudes sociales, base de la auténtica participación comunitaria.

${ }^{12}$ Cfr. DE LANDESHEERE, G., La investigación educativa en el mundo., p. 382.

13 Virtud es el hábito bueno, adquirido por repetición de actos y que proporciona facilidad y agrado en el actuar. 
El planteamiento global está integrado aun en zonas aparentemente marginadas, pues los medios de difusión acercan lo lejano, borran las distancias e informan de sucesos en lugares antes desconocidos. Sin embargo, el peligro de tanta apertura puede darse en personas con escasa educación o con algún tipo de marginación local, entonces pueden evadirse de las responsabilidades inmediatas y soñar con lo lejano en donde sólo mediáticamente pueden participar. Así, viven de ensueños poco productivos.

La participación en la vida comunitaria no es solamente una de las mayores aspiraciones del ciudadano - llamado a ejercitar libre y responsablemente el propio papel cívico con y para los demás-, sino también uno de los pilares de todos los ordenamientos democráticos, además de una de las mejores garantías de permanencia de la democracia ${ }^{14}$.

En el Congreso «Humanismo solidario», realizado en Cuernavaca, Morelos, en abril de 2007, se concretaron catorce propuestas ${ }^{15}$ : En ellas se descubren las inquietudes de participación y de obtener de cada persona lo mejor de sí:

$1^{a}$. Elegir estrategias adecuadas para aplicar un criterio humanista solidario.

$2^{\mathrm{a}}$. Fundamentar en planteamientos éticos la credibilidad del sistema político.

$3^{\mathrm{a}}$. Asegurar la transparencia como base para lograr un auténtico diálogo entre el gobierno y la sociedad. El gobierno ha de tener una actitud de escucha respetuosa, la sociedad ha de hacerse oír con oportunidad para ser tomada en cuenta.

${ }_{14}$ Cfr. Compendio de la doctrina social de la Iglesia., n. 190.

15 Cfr. Boletín msmg. 
$4^{a}$. Crecer en responsabilidad frente a la democracia electoral para conseguir la democracia participativa.

$5^{\text {a }}$. Lograr la participación ciudadana en el sistema político para obtener la reforma sana del Estado.

$6^{a}$. Practicar la vertebración cívica del humanismo solidario para establecer la mejora continua del binomio mejor sociedad/mejor gobierno.

$7^{\mathrm{a}}$. Establecer una política de Estado que articule gobierno y sociedad para erradicar la miseria.

8. Ampliar la productividad y el empleo como un compromiso prioritario.

$9^{a}$. Elegir un sistema educativo que logre resultados concretos en cada ciudadano.

$10^{\mathrm{a}}$. Aplicar un sistema de justicia sólido y completo para mejorar la seguridad.

$11^{a}$. Garantizar la aplicación de la ley para contrarrestar el crimen organizado.

$12^{\mathrm{a}}$. Contar con la participación de todos los miembros de la sociedad para asegurar el desarrollo sustentable.

$13^{\mathrm{a}}$. Estudiar una reforma laboral que vele por el equilibrio de intereses para alcanzar una sana competitividad.

$14^{a}$. Conseguir la colaboración entre ciudadanos y gobierno para asegurar el futuro adecuado de la población con el acceso de todos al bien común.

Obviamente cada uno de estos puntos se facilitará si se aplican los valores personales y los relacionales. Además, dos virtudes son imprescindibles: la prudencia y la justicia. La prudencia para elegir el mejor momento para poner en práctica cada propuesta, previa selección de las 
más urgentes. Además, esta virtud aconsejará sobre quién se hará cargo de cada proyecto y cómo se realizarán. La prudencia siempre se ha visto necesaria en los gobernantes.

La justicia que consiste en dar a cada quien lo debido, es fuente de paz. La injusticia provoca comparaciones, insatisfacción y violencia porque incita a conseguir lo que cada uno merece... pero por su propia cuenta. Así también se produce desorden, pues cada quien toma la justicia por su mano. La justicia está profundamente vinculada al respeto. La justicia evita el egoísmo y la avaricia.

\section{PROPUESTA}

Para facilitar a los sistemas educativos la focalización de objetivos en valores y en virtudes, a continuación se presenta un cuadro donde se seleccionan los que podrían ser más inmediatos. Esta simplificación busca facilitar la tarea pero de ninguna manera reduce la amplísima gama de valores y de virtudes. Sin embargo, al impulsar algunos se impulsan indirectamente los demás.

\begin{tabular}{|l|l|l|}
\hline \multicolumn{1}{|c|}{ PROCESO } & VALORES & VIRTUDES \\
\hline $\begin{array}{l}\text { Elegir estrategias adecuadas para } \\
\text { aplicar un criterio humanista solidario. }\end{array}$ & $\begin{array}{l}\text { Libertad } \\
\text { Solidaridad }\end{array}$ & Prudencia \\
\hline $\begin{array}{l}\text { Fundamentar en planteamientos éticos } \\
\text { la credibilidad del sistema político. }\end{array}$ & $\begin{array}{l}\text { Intimidad } \\
\text { Lealtad }\end{array}$ & Prudencia \\
\hline $\begin{array}{l}\text { Asegurar la transparencia como base para un } \\
\text { diálogo auténtico entre el gobierno y la sociedad. } \\
\begin{array}{l}\text { El gobierno ha de tener una actitud de escucha } \\
\text { respetuosa; la sociedad ha de hacerse oír con } \\
\text { oportunidad para ser tomada en cuenta. }\end{array}\end{array}$ & Comunicabilidad & Prudencia \\
\hline $\begin{array}{l}\text { Crecer en responsabilidad frente a la } \\
\text { democracia electoral para conseguir } \\
\text { una democracia participativa. }\end{array}$ & Respeto & Justicia \\
\hline
\end{tabular}




\begin{tabular}{|l|l|l|}
\hline \multicolumn{1}{|c|}{ PROCESO } & VALORES & VIRTUDES \\
\hline $\begin{array}{l}\text { Lograr la participación ciudadana en } \\
\text { el sistema político para conseguir la } \\
\text { reforma sana del Estado. }\end{array}$ & Solidaridad & $\begin{array}{l}\text { Prudencia } \\
\text { Justicia }\end{array}$ \\
\hline $\begin{array}{l}\text { Practicar la vertebración cívica del humanismo } \\
\text { solidario para establecer la mejora continua } \\
\text { del binomio mejor sociedad/mejor gobierno. }\end{array}$ & Solidaridad & Justicia \\
\hline $\begin{array}{l}\text { Establecer una política de Estado que articule } \\
\text { gobierno y sociedad a fin de erradicar la miseria }\end{array}$ & Subsidiariedad & Justicia \\
\hline $\begin{array}{l}\text { Ampliar la productividad y el empleo como } \\
\text { un compromiso prioritario. }\end{array}$ & Solidaridad & Justicia \\
\hline $\begin{array}{l}\text { Elegir un sistema educativo que logre } \\
\text { resultados concretos en cada ciudadano. }\end{array}$ & $\begin{array}{l}\text { Comunicabilidad } \\
\text { Respeto }\end{array}$ & $\begin{array}{l}\text { Prudencia } \\
\text { Justicia }\end{array}$ \\
\hline $\begin{array}{l}\text { Aplicar un sistema de justicia sólido } \\
\text { y completo para mejorar la seguridad. }\end{array}$ & Respeto & Justicia \\
\hline $\begin{array}{l}\text { Garantizar la aplicación de la ley para } \\
\text { contrarrestar el crimen organizado. }\end{array}$ & Respeto \\
\hline $\begin{array}{l}\text { Contar con la participación de todos } \\
\text { los miembros de la sociedad para asegurar } \\
\text { el desarrollo sustentable. }\end{array}$ & Solidaridad & Justicia \\
\hline $\begin{array}{l}\text { Estudiar una reforma laboral que vele por } \\
\text { el equilibrio de intereses para alcanzar } \\
\text { una sana competitividad. }\end{array}$ & $\begin{array}{l}\text { Solidaridad } \\
\text { Respeto }\end{array}$ & Pubsidiariedad \\
\hline $\begin{array}{l}\text { Conseguir la colaboración entre ciudadanos y } \\
\text { gobierno para asegurar el futuro adecuado de la } \\
\text { población con el acceso de todos al bien común. }\end{array}$ & $\begin{array}{l}\text { Prudencia } \\
\text { Justicia }\end{array}$ \\
\hline
\end{tabular}




\section{BIBLIOGRAFÍA}

LANDESHEERE, Gilbert de., La investigación educativa en el mundo., $3^{\text {a }}$ reimpresión., Fondo de Cultura Económica., México., 2003.

LÓPEZ DE LLERGO, Ana Teresa., Valores., valoraciones y virtudes., CECSA., Patria Cultural., México., 2000.

LÓPEZ DE LLERGO, Ana Teresa., Educación en valores, educación en virtudes., CECSA., Patria Cultural., México., 2003.

LÓPEZ DE LLERGO, Ana Teresa y CRUZ DE GALINDO, Luz María., «Desarrollo comunitario y calidad de vida»., Revista Panamericana de Pedagogía. Saberes y quehaceres del pedagogo., n. 4., Facultad de Pedagogía., Universidad Panamericana., México., enero., 2003.

PÉREZ ADÁN, José., «La comunidad educativa»., en NAVAL, Concepción y LASPALAS, Javier (coords.)., La educación cívica hoy. Una experiencia interdisciplinar., EUNSA., Pamplona., 2000.

POLAINO-LORENTE, Aquilino y MARTÍNEZ CANO, Pedro., La comunicación en la pareja., Ediciones Rialp., Madrid., 1999.

RATZINGER, Joseph., Verdad, valores, poder., Ediciones Rialp., Madrid., 1995.

YEPES STORK, Ricardo y ARANGUREN ECHEVARRÍA, Javier., Fundamentos de Antropología. Un ideal de la excelencia humana., $3^{\text {a }}$ edición., EUNSA., Pamplona., 1998.

MEJOR SOCIEDAD MEJOR GOBIERNO., Boletín MSMG [en línea]., 2 de junio 2007. En: [http: / / www.msmg.org.mx/boletin/ historico/b52msmg.htm].

Compendio de la doctrina social de la Iglesia., Ediciones CEM/Conferencia del Episcopado Mexicano., México., 2005. 